\title{
LA CONSTRUCCIÓN DE SUJETOS EN TORNO A LA INSTITUCIONALIZACIÓN DE POLÍTICAS PÚBLICAS DE GÉNERO EN CHILE
}

\section{THE CONSTRUCTION OF SUBJECTS AROUND IN THE PROCESSES OF INSTITUTIONALIZATION OF PUBLIC GENDER POLICIES IN CHILE}

\author{
Paulina Orellana. Facultad Latinoamericana de Ciencias Sociales - Ecuador \\ pauliorellanac@gmail.com
}

Resumen Este artículo analiza cómo se construyen sujetos por parte del Estado en torno a la institucionalización de políticas de género en un contexto neoliberal ${ }^{1}$. Mediante una metodología cualitativa y el método etnográfico, se realiza un análisis de documentación histórica y bibliografía secundaria referida al movimiento feminista chileno "Concertación Nacional de Mujeres por la Democracia". Lo anterior, permite desentramar desde los condicionamientos estructurales del neoliberalismo y las relaciones entre Estado y política pública cómo se articularon las demandas del movimiento feminista con la creación del Servicio Nacional de la Mujer en 1991.

Palabras clave Institucionalidad, políticas públicas, género, Estado neoliberal, construcción de sujetos.

\begin{abstract}
This article analyzes how is the State's construction of subjects around Gender Policies institutionalization on a Neoliberal context. Through a qualitative and ethnographic method, it presents a historical and secondary data collection to analyze Chilean feminist movement "Concentración Nacional de Mujeres por la Democracia". This allows to unravel how feminist movement's demands were articulate with Neoliberalism structural conditioning and the relationship between State and public policy, with the creation of Servicio Nacional de la Mujer on 1991.
\end{abstract}

Keywords Institutionality, Public Policies, Gender, Neoliberal State, subject construction

\section{Introducción}

En agosto de 1983 un grupo de mujeres extiende un lienzo en las escalinatas de la Biblioteca Nacional, con la consigna "Democracia Ahora. Movimiento Feminista". Instaurándose este hito como la primera actividad pública del movimiento feminista de Chile durante la dictadura militar, régimen que gobernó el país desde el año 1973 hasta 1989.

Luego, en enero de 1991 bajo el gobierno de Patricio Aylwin, y como parte del compromiso adquirido por los partidos políticos contrarios a la dictadura militar con el

\footnotetext{
${ }^{1}$ Sustentada en mi investigación de maestría "La paradoja entre los discursos de igualdad y las prácticas desiguales. La construcción de sujetos en torno al género en Chile: El caso del Servicio Nacional de la Mujer".
} 
movimiento feminista, se crea el "SERNAM", agencia técnica del Estado que llevaría a cabo la implementación de políticas públicas de género. Lo anterior se posiciona como el más grande triunfo del movimiento, cuyo horizonte se basaba en producir transformaciones sociales, culturales y políticas y la visibilización de las diversas esferas de desigualdad que vivían las mujeres en Chile.

Sobre lo anterior y entendiendo que los procesos locales se anclan en procesos globales, este artículo problematiza en torno a la institucionalización de políticas de género en Latinoamérica y el contexto social y económico de Chile en particular, para analizar el papel de los Estados en las relaciones de género, los actores sociales en la negociación de políticas públicas de género, los movimientos feministas y de mujeres en la región y la institucionalización de políticas de género en el contexto neoliberal.

Este artículo comienza mediante una contextualización teórica sobre la construcción de sujetos en torno al género en el Estado neoliberal y la institucionalización de políticas de género. El acápite del desarrollo, se refiere al contexto latinoamericano y los movimientos feministas de los años 80 y 90, y profundiza puntualmente en el movimiento feminista en Chile y su facción "CNMD" y la institucionalización de las demandas de género. Finalmente, el artículo cierra a través de reflexiones y conclusiones, que darán cuenta de qué sujetos se construyen en torno a las políticas de género, cómo se abordaron las demandas del movimiento feminista y cómo se articuló esto con el sistema económico del país.

La importancia del análisis de este momento histórico, hito en cuando a la institucionalidad de género en Chile, es que da cuenta de una instancia atravesada por paradojas, donde se plantean medidas desde el Estado para abordar las desigualdades en torno al género mediante la institucionalización de políticas públicas de este orden y la creación de una institución para estos fines.

\section{Metodología}

La investigación se abordó desde una metodología cualitativa a través del método etnográfico, mediante técnicas como análisis de texto y análisis de bibliografía secundaria. Se realiza una imbricación analítica sobre el análisis de documentación histórica relativa a resoluciones oficiales de gobierno, cartas y actas de trabajo de la "CNMD" retomadas del portal virtual de la Universidad Alberto Hurtado en Chile ${ }^{2}$ y de bibliografía secundaria que se refiere al movimiento en sí y a la forma en que las demandas feministas fueron institucionalizadas en el gobierno postdictadura de Aylwin con la creación del "SERNAM". Sobre esto último, se utilizarán informes trimestrales y anual de gestión de la institución para el año 1991, para efectos de ahondar en los programas establecidos y el enfoque institucional en cuanto el abordaje de las desigualdades sociales en relación al género.

\footnotetext{
${ }^{2}$ http://archivospublicos.cl/
} 


\section{Marco teórico}

\subsection{El Estado neoliberal y la construcción de sujetos en torno al género}

Para revisar el momento histórico propuesto, es necesario analizar desde distintas entradas teóricas que nos permitan problematizar en tono al carácter heterogéneo, dinámico y conflictivo del Estado en el neoliberalismo (Bourdieu, 1997; Foucault, 1999-2007; Harvey, 2007; Wacquant, 2013), la institucionalización de políticas públicas de género y la conceptualización de un sujeto mujer (Beneria y Sen, 1982; Fraser, 1989-2015; Guzmán, 1997; Girón, 2009; Schild, 2000-2015-2016).

Esta imbricación teórica permite cuestionar qué tipo de sujetos se construyen en torno en las políticas de género y cómo se construye un sujeto mujer de intervención en relación con lo presentado por algunas autoras que se enmarcan desde la teoría feminista y los estudios de género (Beneria, 1999; Anzaldúa, 1999; Lorde, 2002; Mahmood, 2008; Molyneux, 2008; Hill Collins, 2012) en torno al reconocimiento de las distintas necesidades de las mujeres y los procesos de exclusión social, marcados por desigualdades en torno a la raza, clase, religión, sexualidad, contexto social y político y la naturalización de roles.

\subsection{Neoliberalismo, desigualdades sociales y la construcción del sujeto neoliberal}

Desde las ciencias sociales se han planteado numerosos debates sobre las diversas reestructuraciones sociales, políticas, económicas y culturales que trae aparejado el actual momento del sistema productivo capitalista: el neoliberalismo económico, y el reforzamiento de viejas y nuevas desigualdades sociales. Los debates permiten analizar las transformaciones de las subjetividades, de las identidades nacionales, las desigualdades en el sistema productivo, la tercerización y flexibilización del mercado laboral, las desigualdades de género, entre otros tópicos, elementos que resultan clave a la hora de pensar la relación entre el Estado, el neoliberalismo y las desigualdades sociales.

Harvey (2007) define al neoliberalismo como una teoría de las prácticas políticoeconómicas, donde se sostiene que la mejor manera de promover el bienestar de las personas, es no limitar el libre desarrollo de las capacidades y de las libertades empresariales, contexto enmarcado por el fortalecimiento de los derechos de propiedad privada y robustos mercados libres donde el Estado cumple con crear el marco institucional para que estas prácticas político-económicas se den de forma apropiada.

Desde la década del 1970 todos los Estados neoliberales han sostenido un proceso de desregulación y privatización y han abandonado paulatinamente las acciones destinadas a la provisión social, por lo que el neoliberalismo ha desmontado el Estado 
de bienestar, frenando la inversión social y aumentando cada vez más las políticas en favor de las relaciones de mercado. Prácticamente todos los Estados, de manera voluntaria o bajo la presión internacional, han ajustado sus políticas a las premisas neoliberales, ideología que se torna hegemónica no sólo en lo económico, sino también en lo discursivo (Harvey, 2007).

Destaca Murillo (2011) que la implantación del neoliberalismo a mediados de los años 70 en Europa y Latinoamérica, modificó el abordaje de las políticas públicas de los gobiernos hacia las poblaciones, de igual forma se reconfiguran los roles y significados otorgados al Estado y al mercado y se reestructuran los procesos de subjetivación.

Por su parte Foucault (2007), indica que los procesos de individualización y libertad como parte fundamental del modelo liberal y de los modelos de subjetividad tienen un papel hegemónico en el plano económico y político. Sostiene que, en el liberalismo se acusó el poder de los Estados en cuanto a la regulación económica, con la tesis de que se constituían como el principal impedimento que trababa la exitosa autorregulación de los mercados. En efecto, el neoliberalismo retoma esta premisa y sitúa a la desregulación, la competición y la libertad como los nudos centrales que orientan las prácticas económicas y sociales, otorgando a estas categorías el valor de inalienables y como parte intrínseca de la subjetividad individual, que entiende las relaciones sociales como un terreno de juego donde competir es una necesidad.

Sobre esto, las prácticas neoliberales se ven también sustentadas y reguladas en el marco institucional que provee el Estado, donde Foucault (2007) reconoce que la razón gubernamental neoliberal permite la multiplicación del modelo económico al individuo, la propiedad privada y la familia. Así, el neoliberalismo se convierte en el actual instrumento del capitalismo que organiza la acción de las autoridades, la conducta y subjetividades de los individuos.

En relación a la construcción de sujetos en el neoliberalismo, el discurso neoliberal se torna hegemónico no sólo en el sentido económico y de mercado, sino que además en las formas en que los gobiernos neoliberales moldean y conducen a los sujetos (Murillo, 2011).

Bauman (1999) sostiene que en la actualidad las subjetividades de los individuos se mueven en torno a la concepción de "sujeto consumidor", condicionada por la sociedad de consumo actual, contexto marcado por la constante necesidad de satisfacer múltiples necesidades y la búsqueda de felicidad en la concreción de satisfacción. En definitiva, las subjetividades están marcadas por la creación de sujetos dependientes y desiguales en un proceso cada vez más fuerte de dualización de la sociedad, con un sector de élite y una mayoría desempleada o precarizada (Rodríguez, 2003).

\subsection{Estado neoliberal, género y políticas públicas}


Pensar el neoliberalismo nos remite obligatoriamente a pensar el rol del Estado frente a este. Desde la teoría social diversos enfoques lo posicionan en distintos espacios dentro del proceso. Por un lado y tal como ya revisamos, Harvey (2007) sostiene que el Estado tiene el rol de crear el marco institucional apropiado para el ejercicio de las prácticas político-económicas.

Bourdieu (1997) desde el concepto de campo burocrático, define al Estado como un aparato de estratificación y clasificación, consecuencia de un proceso de acumulación de distintos tipos de capital: capital simbólico, capital de fuerza física, capital económico y capital cultural. Esta acumulación de capitales establece al Estado como poseedor de un capital propiamente estatal. También destaca que la construcción del Estado, va aparejado con la construcción de un campo de poder en donde los detentores de capital luchan por el poder del Estado. Reconoce además que uno de los grandes poderes que posee el Estado es el de producir e inculcar categorías de pensamiento y abstracción que aplican los individuos de forma subjetiva a cualquier cosa del mundo y al Estado mismo, bajo la forma de estructuras mentales.

Bourdieu (1997) destaca que para pensar el Estado hay que cuestionar todas las preconcepciones que están inscritas en la realidad que se quiere analizar. Así, es importante tener presente que el Estado se hace sentir principalmente en el control de la producción simbólica, en dónde la administración pública y quienes forman parte de ésta, son generadores de múltiples problemáticas y desigualdades sociales. Por tanto, mediante el capital simbólico se disponen las formas para implantar esquemas simbólicos perdurables.

Siguiendo a Bourdieu, Wacquant (2013) sostiene que en el neoliberalismo ocurre un rediseño y redespliegue del Estado como un actor central que impone leyes y constructor de subjetividades, relaciones sociales y representaciones colectivas para hacer realidad los mercados. El Estado "conduce la revolución neoliberal desde arriba, interpretando así el neoliberalismo como una articulación del Estado, el mercado y la ciudadanía, que emplea al primero para imponer el sello del segundo en el tercero" (Wacquant, 2013:1).

Wacquant (2013) indica que el concepto de campo burocrático, nos ofrece una herramienta analítica para pensar al Estado como un aparato de estratificación que conduce el proceso neoliberalizador, en donde el neoliberalismo no es sólo un proyecto económico sino también político, por lo que no implica el desmantelamiento del Estado sino su reconstrucción.

Otro elemento importante de problematizar en torno a las relaciones de poder que confluyen en el Estado, para eso retomaremos el concepto de gubernamentalidad de Foucault (1999). La gubernamentalidad proviene de lo que el autor entiende como saber-poder y se centra en las relaciones entre sujetos, dónde el poder está en el centro de las relaciones sociales. Para Foucault, el Estado se instaura como consecuencia de la articulación de diversas tecnologías orientadas a modelar la conducta de los individuos. En lo que enfatiza el autor es en la forma en que el 
liberalismo y el neoliberalismo producen condiciones o marcos de aceptabilidad en donde los sujetos se perciben como individuos autónomos, a pesar de que los propósitos de sus conductas sean dirigidos por quienes detentan el poder

El término gubernamentalidad, por tanto, se refiere a un régimen de poder dirigido a la población, encabezado por el gobierno. Es una tecnología general de poder que incluye el propio autocontrol y el control de las poblaciones, por lo que define las formas, condiciones y el tipo de gobierno en un momento o contexto específico.

\subsection{El género en el Estado neoliberal y la problematización del sujeto "mujer"}

Hasta este punto hemos visto qué Estado se construye en el neoliberalismo y las relaciones de poder que confluyen en él, por lo que falta problematizar aún el posicionamiento del Estado neoliberal frente al género. Al respecto, vemos mediante el ejemplo de Latinoamérica, cómo las agendas, discursos y prácticas feministas de las últimas décadas han condicionado un importante cambio en los Estados reducidos por las reformas estructurales que trajo la implantación del neoliberalismo en las décadas de los 70 y 80 , donde el análisis feminista se centra en problematizar en torno el legado feminista y su relación con el "estado social" en el momento actual (Schild, 2015).

Enfatiza Schild (2015) que a nivel internacional fracasaron muchas de las políticas propuestas bajo el Consenso de Washington, por lo que a fines de los noventa se promueven estrategias de cambio que buscan rescatar el rol del Estado en el desarrollo, impulsando políticas centradas en los problemas sociales. Lo interesante de este análisis, es que retoma una discusión que esta escasamente presente y desarrollada en los debates sobre el Estado social y que se refiere a la dimensión de género en la construcción del Estado social neoliberal.

Es importante puntualizar que el neoliberalismo condiciona la creación de un sujeto neoliberal, en dónde el Estado juega un papel trascendental a la hora de interiorizar en los individuos las premisas neoliberales. Tal como se adelantó, Schild (2015) analiza cómo la inclusión al ámbito público de las demandas feministas ocurrió en un momento en que las administraciones públicas de Latinoamérica llevaban a cabo importantes procesos de modernización y reestructuración del Estado fomentados por el Banco Mundial, que buscaban rescatar la noción de un Estado capaz y eficaz, pero bajo la lógica del sector privado.

Así, uno de ámbitos en el que el Estado neoliberal cobra vigencia, es en la protección social mediante programas de alivio y superación de la pobreza, en los que se busca convertir a los beneficiarios en personas que sean capaces de hacerse responsables de su propio desarrollo, en ese sentido, el proyecto neoliberal se basa en adaptar a los individuos al modelo de acumulación actual, fortaleciendo las 
capacidades personales y de emprendimiento para insertarse exitosamente al mercado.

El énfasis en la autonomía individual y el reforzamiento de las capacidades económicas de los individuos trae al escenario político y social a las mujeres como pieza fundamental, configurándolas como usuarias o consumidoras de programas estatales, encargadas de apartar a sus familias de la pobreza, pero también como ejecutoras de estos programas, en donde la lógica del capitalismo flexibilizado propició la apertura de oportunidades laborales para las mujeres, pero con las características típicas del trabajo femenino, precarios y mal pagados. Si bien, como se revisó en las características del neoliberalismo la precarización laboral no es indiferente a ningún sexo, las mujeres enfrentan un tipo de discriminación adicional justificada en el género, no en vano son las que engruesan las filas de trabajadores mal pagados y precarios del ámbito formal (Schild, 2000).

Finalmente indica la autora, que la inclusión de las mujeres a las políticas públicas, ya sea como usuarias o ejecutoras no problematiza las situaciones de desigualdad en las que están inmersas. No cuestiona tampoco cómo se afianzan los roles al pensar que las mujeres son las encargadas de sacar a sus familias de la pobreza y se las visibiliza como sujeto económico y no como sujeto político, instalando la reproducción social de la vida como el punto central de sus necesidades.

El Estado, por tanto, se entiende como una instancia que permite relaciones de género asimétricas mediante políticas y programas destinados a la previsión social. En ese sentido, el acceso de las mujeres a derechos sociales está circunscrito a su situación de madre y/o esposa y a su posición dentro del mercado laboral, instancia en las que generalmente cumplen labores como mano de obra informal, flexible, precarizada y con bajas remuneraciones (Molyneux, 2008).

Para Fraser (1997), existe una estructura de género en la economía política que produce las desigualdades en torno al género, lo que genera la división entre trabajo productivo y trabajo doméstico y que también estructura la división en el trabajo pagado, lo que se traduce en que las ocupaciones industriales y profesionales mejor remuneradas son ocupadas principalmente por hombres, mientras que las mujeres ocupan aquellas actividades de "cuello rosa" (Fraser, 1997:138).

Lo desarrollado anteriormente, nos da pistas analíticas para problematizar cómo se piensa a las mujeres y sus necesidades desde el Estado neoliberal, e interpela a ahondar sobre la concepción de género y cómo se piensan las políticas públicas de género. Temas que las teorías feministas y los estudios de género han discutido largamente.

Para una definición de género, Scott (2008) plantea que es un elemento constitutivo de las relaciones sociales, basadas en las diferencias percibidas entre los sexos, con cuatro elementos constitutivos: los símbolos; los conceptos reguladores o normativos; instituciones sociales y la identidad subjetiva. También sostiene que el género es una forma elemental de las relaciones simbólicas de poder, dentro del cual 
o por medio del cual este se articula. Esto permite entender cómo el género ha sido recurrentemente una esfera donde el poder político ha sido criticado, legitimado y concebido. Por lo tanto, el hecho de cuestionar o alterar las relaciones de género, representa una amenaza para el sistema.

Por su parte, Argüello (2013) reconoce cómo los feminismos han cuestionado las premisas naturalistas de adjudicación de roles y actividades femeninas y masculinas y el otorgamiento de espacios diferenciados a hombres y mujeres. Han problematizado también la forma en que se reproducen las relaciones de poder, explotación y subordinación de forma distintiva por sexo y mediante la categoría "género" han visibilizado cómo ciertos rasgos naturales responden a construcciones culturales, por lo que ser hombre o mujer "pasa por comprender los entramados sociales, culturales e históricos de las relaciones de poder entre los sexos y las definiciones sociales acerca de lo que se acepta o no en torno al sexo y al género" (Argüello, 2013:5).

En ese sentido, los feminismos han desarrollado diversos debates que plantean el reconocimiento de las relaciones de poder, desigualdad y la subjetividad en la interpretación social, generando así importantes discusiones en torno a temáticas como la división público/privado, la articulación entre producción capitalista y reproducción social de la vida, los procesos identitarios y de identificación, la construcción del sujeto colectivo, la justicia social y las desigualdades.

A fines de los 40, Simone de Beauvior ([1949] 1999) ya problematizaba en torno a la construcción social del sujeto mujer, bajo la premisa "no se nace mujer: se llega a serlo". Interpelando cómo las mujeres eran catalogadas como un sexo inferior a los hombres. Este precedente sienta las bases para una posterior problematización por parte de diversos feminismos, sobre las concepciones culturales que posicionan a las mujeres en una situación desventajosa dentro de la sociedad y también reconociendo las especificidades de estas de acuerdo a variables como clase, raza, religión, contexto social y político, entre otros. Esto también permitió cuestionar la categoría mujer cómo única y particular (Beneria, 1999; Anzaldúa, 1999; Lorde, 2002; Mahmood, 2008; Molyneux, 2008; Hill Collins, 2012).

Si bien, desde diversos feminismos se ha abordado lo problemático que es reducir la categoría mujer y sus necesidades a un sujeto universal, Schild (2015) nos muestra, cómo desde la acción estatal en el contexto neoliberal, se generalizan las necesidades de las mujeres en la figura de una mujer pobre, que debe sacar a su familia de la pobreza y que necesita del Estado para insertarse en el mercado.

Este punto nos lleva a problematizar la forma en que la acción estatal se hace sentir en la población y que es mediante políticas públicas.

\subsection{Políticas públicas y la construcción del sujeto mujer}


Wappenstein y Villamediana (2014), reconocen que la inclusión del género cómo categoría de análisis en el diseño, implementación y evaluación de políticas en Latinoamérica, ha permitido la identificación de desigualdades recurrentes en las relaciones sociales y se reconocen potenciales áreas de acción en la búsqueda de sociedades menos desiguales. Pero que, a pesar de los esfuerzos de movimientos feministas y de mujeres y de los adelantos a nivel mundial en materia de derechos, las desigualdades sociales en torno al género todavía se reproducen, por lo que se mantiene como un tema que necesita de interés político y de intervención estatal.

Desde los estudios de género, se ha caracterizado a las políticas públicas como un proceso dónde las demandas problemas sociales son transformadas en alternativas políticas y en temas de discusión y resolución de autoridades públicas, necesidades desprendidas de la sociedad civil o de actores políticos (Guzmán, 1997). El curso de este proceso es dinámico y los públicos son construidos por actores que se mueven desde distintos escenarios confrontando discursos desde distintos marcos de sentido. El proceso de la política pública consta de diferentes fases, las cuales pueden ir cambiando de acuerdo a la emergencia de nuevas pautas que condicionen la realidad social (Fraser, 1989).

En ese sentido, Fraser (1989) propone estudiar las demandas de las mujeres como productos de disputas más que las necesidades formuladas en las políticas, disputas que traerían consigo el enfrentamiento de interpretaciones discursivas que muchas veces llevan a despolitizar las necesidades. Así, expone que las políticas de género deben ser entendidas como el reflejo de múltiples intereses de diversos actores inmersos en ellas. Así, (Fraser, 2015) la institucionalización de políticas de género se basa en una política de interpretación de las necesidades por parte del Estado.

Ante esto, Schild (2015) reconoce cómo a partir de década de los 90 podemos hablar de una feminización de los Estados en Latinoamérica, en el sentido de que los programas de género condicionan el empoderamiento familiar, mediante la figura de la madre, que se convierte en la destinataria principal de las políticas de género. De esta forma se sugiere que la construcción del estado neoliberal, en su cara social, es en gran medida un esfuerzo de mujeres y feminista.

Otro punto importante que sostiene Schild (2016), se relaciona con la concepción de sujeto consumidor que se construye desde el Estado neoliberal para la asistencia de las más pobres, que como hemos revisado, se refiere a la inclusión de estas al mercado. Esta categoría deja por fuera entonces las necesidades de las mujeres que no cumplen con los requisitos de focalización que las políticas y programas de género requieren.

En efecto, la construcción neoliberal de un sujeto consumidor, condicionado por políticas de género enfatiza el rol reproductivo de las mujeres y las convierte en clientas de los programas de género, establece jerarquías de clase entre mujeres, crea grupos de mujeres objetivo de políticas y excluye a otras, causando tensiones, clientelismo y exclusión, abandonando espacios colectivos donde las propias mujeres 
puedan definir sus necesidades. En efecto, al no problematizar las situaciones de desigualdad de las mujeres lleva a que se instalen programas de desarrollo simples e incompletos, que no tocan problemas sociales como la explotación de clase y la subordinación de género.

Con respecto a esto, Girón (2009) sostiene que la globalización y el mercado global neoliberal profundizan las desigualdades de género. La globalización condicionó la feminización de la pobreza mediante una articulación estructural que permite una mayor participación laboral de las mujeres, pero un desarrollo desigual, profundizando la inequidad de género y las desigualdades sociales.

Para cerrar, es necesario retomar la noción de justicia de género que plantea Nancy Fraser (2015) y que nos ayudará a entender cuáles son las dimensiones en que las políticas de género deberían actuar y los retos que desde el feminismo se plantean en la búsqueda de una sociedad más justa. Así, la autora propone siete principios normativos que contemplan las distintas situaciones de desigualdad que cruzan las mujeres: el principio de la antipobreza; el principio de antiexplotación; la igualdad de renta; igualdad en el tiempo de ocio; igualdad de respecto; el principio de antimarginación y el principio antiandrocentrista. Fraser recalca que al menos deberíamos aspirar a encontrar métodos que satisfagan los siete principios o al menos la mayoría de ellos, lo que, en pos de esta investigación, nos obliga a pensar como las políticas de género están abordando las dimensiones que la autora reconoce.

El análisis teórico presentado, nos permite ahora situarnos en la experiencia latinoamericana y en específico el chileno y analizar cómo se construyen sujetos en el contexto de la creación del "SERNAM" y el papel de la "CNMD" en la visibilización de las demandas de las mujeres en la dictadura militar.

\section{Desarrollo}

\subsection{Contexto sociopolítico y económico en Latinoamérica y los movimientos feministas y de mujeres}

Los años go se configuran como un período en que los movimientos feministas contemplan al Estado como un interlocutor y espacio válido donde dialogar por sus demandas. Se amplía la perspectiva mantenida por el feminismo en los años 8o, dónde se pensaba al Estado como un espacio homogéneo, donde no se podían negociar las demandas, por concebirlo como un espacio reproductor de desigualdades sociales. En efecto, los 90 se plasman como la década en donde el feminismo comienza a ver el Estado como una institución heterogénea, que necesita ser perfeccionado en su accionar, conciliador de diferencias y como un espacio potencial para la igualdad (Herrera, 2001).

Una de las principales razones para el vuelco de perspectiva sobre el Estado por las feministas, se refiere a la ayuda prestada por estas últimas en el restablecimiento de las democracias en los países de la región que vivieron dictaduras y por la crisis 
económica vivida en la década de los 80 producto de los ajustes estructurales en materia política y financiera por la emergencia del neoliberalismo. Por lo que el feminismo regional comprometió su accionar principalmente a la lucha contra las dictaduras latinoamericanas, el respeto por los derechos humanos y en el respaldo de la transición democrática, en pos de avanzar en el reconocimiento de los derechos de las mujeres (Molyneux, 2008).

En ese sentido, desde los estudios de género (Guzmán, 1997; Álvarez, 1998; Herrera, 2001; Lind, 2005; Molyneux, 2008; Rousseau, 2012) se ha planteado la tarea de analizar el accionar de los movimientos feministas en contextos de dictaduras y de crisis económicas, lo que ayudará a entender el contexto regional y nacional en el que se posiciona el movimiento feminista chileno "CNMD", la institucionalización de políticas de género y la creación del "SERNAM".

En definitiva, el feminismo regional y los movimientos de mujeres se posicionan como principales actores frente a los contextos adversos, condicionando junto a las convenciones internacionales sobre derechos de las mujeres, la institucionalización de las demandas de género. Si bien, esto se convierte en un importante precedente, no debemos olvidar para el abordaje analítico del tema la necesidad de analizar desde el feminismo las concepciones de género que se manejan en la dinámica estatal, para entender las limitaciones que existen a la hora de institucionalizar las demandas feministas. Lo anterior nos plantea el desafío de repensar en qué medida la inserción en el Estado de las demandas de género comprometen las agendas feministas hasta el punto de despolitizar las demandas.

\section{2. "Si la mujer no está, la democracia no va"3}

La lucha de las mujeres en Chile tiene sus antecedentes en la primera mitad del siglo XX, las que luego de ser consideradas en el año 1934 con el derecho a voto en elecciones municipales y en el año 1949 para las elecciones presidenciales y parlamentarias, reaparecen con fuerza luego de casi tres décadas de mutismo, motivado por el contexto sociopolítico dictatorial que imperaba desde 1973.

Para Ríos et. al. (2003) el resurgimiento del feminismo está condicionado por tres elementos, el primero se refiere al desmantelamiento de formas tradicionales de representación ciudadana y de estructuras de partidos políticos y de gobierno, permitiéndose la emergencia de nuevos actores sociales y formas de organización. El segundo factor, se refiere a las políticas y procesos generados en la instalación del régimen militar, caracterizados por la violación de derechos humanos y la grave crisis económica, producto de las políticas de estabilización macroeconómica neoliberales. En efecto, ante la ausencia de partidos políticos y de entidades sociales capaces de enfrentar las consecuencias del modelo económico y político, las mujeres comienzan a organizarse para suplir estas ausencias. Así, fueron estas las que comenzaron a salir a

\footnotetext{
3 Una de las consignas del movimiento feminista en Chile.
} 
la calle a demandar justicia y solicitar información sobre el paradero de sus familiares desaparecidos por la represión militar. El tercer elemento, se relaciona con el discurso de la dictadura, el que exaltaba las diferencias entre los géneros y promovía una visión sesgada de la maternidad y de los roles impuestos a las mujeres, generando un efecto inesperado en estas, puesto que abre espacios para la concientización y ocupación de las mujeres de los espacios públicos. En efecto, el contexto de una extrema polarización ideológica y represión, condiciona la aparición de diversos grupos de mujeres, identificadas con el pensamiento feminista.

Así, en el año 1973 comienza la dictadura militar en Chile y a pesar de que el discurso gubernamental y las políticas apelaban directamente a la lógica de dominación patriarcal (Valenzuela, 1993) empiezan a aparecer las "Organizaciones Económicas Populares", el "Departamento Femenino de la Coordinadora Nacional Sindical" y desde la clandestinidad partidos políticos opositores al régimen dictatorial ayudaron en la organización de grupos de mujeres, condicionando la multiplicación de actividades en distintos sectores sociales.

Cabe destacar, que el régimen militar, contaba con una política dirigida a las mujeres, basada en la concepción tradicional de roles sociales a través de programas llevados a cabo por la Fundación Nacional de Ayuda a la Comunidad, encabezada por Lucia Hiriart, la esposa del dictador. Esta organización coordinaba labores de voluntariado de acción social, bajo un carácter asistencial. También se potenciaban los antiguos Centros de Madres, de los que se eliminó cualquier rasgo de política partidista contraria al régimen, fortaleciéndolos como espacios de integración de la mujer en sus roles de madres/esposas usando el discurso de la moral y la decencia. Quienes presidian estos espacios eran mujeres pertenecientes a la élite nacional y las que desarrollaban labores de voluntariado, eran las mujeres de clase media baja y populares (Godoy, 2013).

A pesar del contexto opresor y restrictivo, en el año 1977 se conforma la primera organización de mujeres que se declara feminista, contraria a la dictadura y se conforman el Centro de Estudios de la Mujer, bajo el apoyo de la Universidad Academia de Humanismo Cristiano. En los años 80 los movimientos sociales de mujeres logran articularse, tener legitimidad, visibilidad y formular una propuesta política, de esa forma surgen articulaciones y organizaciones feministas en todo Chile, tales como el "MEMCH 83" y el "Movimiento Unitario Mujeres por la Vida". El movimiento feminista logró posicionarse como una organización amplia, diversa y pluriclasista y su emblema "Democracia en el país y en la casa" ${ }^{4}$, traspasó las fronteras, instalándose en el mundo como la demanda de las mujeres chilenas (Valdés, 1987).

\footnotetext{
${ }_{4}^{4}$ Consigna ideada por Julieta Kirkwood, no sólo asumida por el movimiento de mujeres en Chile sino también por movimientos feministas latinoamericanos.
} 
La primera actividad pública del movimiento feminista en dictadura, se realiza en agosto de 1983, cuando un grupo de mujeres extiende un lienzo en las escalinatas de la Biblioteca Nacional, con el lema: "Democracia Ahora. Movimiento Feminista". De esta forma el movimiento de la década de los 80 fue construyendo una agenda de trabajo que unificaba a las mujeres, en dónde las pobladoras de sectores populares ejercían el rol principal de alimentar a la población pobre mediante la organización de ollas comunes y concientizar sobre de la necesidad de retornar a una democracia (Ríos et.al., 2003).

Así, la agenda de trabajo, se hacía cargo de denunciar la violencia de género, la discriminación y la exclusión de las mujeres del plano político y económico. El movimiento feminista apoyó la derogación de la dictadura bajo la posibilidad de que en un próximo gobierno democrático se les dieran los espacios necesarios para reivindicar la igualdad, autonomía y justicia.

\subsection{La "CNMD" y la creación del "SERNAM"}

La institucionalización del régimen militar se sustenta con la imposición de la Constitución de 1980. Mediante esta, la Junta Militar intentó asegurar el paso de un régimen militar a uno autoritario, para lo cual se establece que en 1988 se someterá a Plebiscito el nombre de un candidato a Presidente para el período 1989-1997, siendo el nombre de Augusto Pinochet el elegido para este propósito (Garretón, 1988).

Así el 5 de octubre de 1988 mediante la realización del Plebiscito del SI o NO, la ciudadanía opta ponerle fin a la dictadura y dar paso a elecciones democráticas. La votación femenina en el Plebiscito demostró cuan fructífera había sido la labor del movimiento de mujeres 5 , y aun cuando las organizaciones de mujeres reconocían en las prácticas políticas alternativas un espacio dónde podían trabajar por mejorar sus situaciones de desigualdad, igualmente se daban cuenta de la necesidad de promover políticas de género mediante espacios convencionales, sobre todo por la eminente vuelta a la democracia y así presionar por la incorporación de los temas de las mujeres en la agenda política.

Frente a esto, el movimiento dividió su accionar en dos tendencias: la "Coordinadora de Organizaciones Sociales de Mujeres" continuó promoviendo el rol de las organizaciones sociales y la necesidad de su participación directa; otro sector de mujeres, militantes de partidos y profesionales se agruparon en la "CNMD", la que se abocó en apoyar a la Concertación de Partidos por la Democracia elaborando el Programa de las Mujeres, que debería ser incorporado en el gobierno democrático, referido principalmente al reconocimiento de las mujeres como sujetos políticos, que se les reconocieran sus derechos sexuales y reproductivos, que se crearán programas

\footnotetext{
${ }^{5}$ Extraído de: http://www.archivospublicos.cl/index.php/memorandum-las-mujeres-frente-al-plebiscito

${ }^{6}$ Extraído de: http://archivospublicos.cl/index.php/que-es-la-concertacion-nacional-de-mujeres-por-lademocracia
} 
en contra de la violencia y que se subsanara la situación de pobreza y dependencia económica que estas sufrían (Montecinos y Rossetti, 1990; Baldez, 1999).

La facción "CNMD", mantuvo un importante papel público y político en el proceso de la redemocracia7. Podemos destacar que se trata de una organización de vocación masiva, con la finalidad de integrar en su quehacer a todas las mujeres, interesadas en trabajar por los derechos de la mujer, por la democracia y por descentralizar la acción del Estado a todos los rincones del país. De la misma forma hacen énfasis en que a pesar del contexto adverso de dictadura, las mujeres han participado activamente en la vuelta a la democracia.

Considerando que son mujeres pertenecientes a partidos políticos, se plantean como objetivo el definir políticas para subsanar las desigualdades sociales y económicas que sufren las mujeres y que deben llevarse a cabo en el gobierno democrático de Patricio Aylwin. A través de la introducción de las problemáticas de las mujeres a la agenda estatal, esperan además promover figuras femeninas en la política y la administración pública, buscar las estrategias para mostrar a la sociedad chilena las problemáticas de las mujeres, crear un lenguaje político que les permita sensibilizar a todas las mujeres del país y velar para que el trabajo político que se realice en vías a la campaña presidencial postdictadura de amplia cabida al debate respecto a los derechos de las mujeres.

Para lograr sus propósitos, la "CNMD" realiza un arduo trabajo de concientización con las mujeres, mediante declaraciones públicas y la circulación en medios escritos de comunicación de imágenes con contenido alusivo a la importancia de apoyar un futuro gobierno democrático. Así, instan a las mujeres a trabajar unidas para lograr la mayor votación posible para el candidato Aylwin. Otra acción, fue realizar diversas encuestas y entrevistas a mujeres en el país, para transmitir al candidato presidencial por la democracia lo que las mujeres quieren y necesitan, ejercicio que permitió sondear que las necesidades van desde el reconocimiento básico como seres humanos, subsanar las desigualdades en materia de empleos y acceso a recursos económicos, hasta el reconocimiento de sus derechos sexuales y reproductivos (Montecinos y Rossetti, 1990; Godoy, 2013; Baldez, 1999; Valdés y Fernández, 2006; Schild, 2015-2016).

Producto del escenario que establecen las convenciones internacionales en torno a las desigualdades de género y la negociación del movimiento feminista con el gobierno democrático de Aylwin, se crea el 3 de enero de 1991 el "SERNAM", organismo del Estado de Chile destinado a promover en la sociedad la igualdad, 
autonomía, equidad, no discriminación y una vida libre de violencia para las mujeres y la implementación de políticas que transversalicen la equidad de género en el Estado ${ }^{8}$.

En ese mismo sentido, Guzmán y Montaño (2012) indican que en Chile el "SERNAM" se crea en respuesta a las demandas formuladas por la "CNMD". El gobierno de Aylwin, en negociación con los partidos políticos de la Concertación y los distintos grupos y actores que resistieron a la dictadura, es sensible a las demandas de las mujeres, incorporando en los lineamientos del programa de gobierno, la igualdad entre mujeres y hombres y creando una institucionalidad pertinente en respuesta a las problemáticas sociales movilizadas. En ese sentido, cuestiono lo anterior, puesto que como revisaremos, las demandas feministas no fueron del todo incorporadas.

Para adelantar el escenario y dar pistas analíticas de las paradojas en la creación del "SERNAM", es necesario retomar la siguiente cita:

"nos aproximamos a este proyecto de ley con un criterio y con una exigencia compartidos, en cuanto a que el ámbito de lo privado, de lo individual y de lo familiar no puede ser interferido por acciones gubernamentales. Esos son, por lo demás, la posición oficial del Gobierno y el espíritu y la letra del proyecto de creación del SERNAM. (...) No hay detrás de esta iniciativa legal determinada concepción ideológica sustentada en una visión parcial del feminismo, sobre el cual existen diversas posiciones. Esperamos compartir con quienes conformamos este Senado una aproximación ética y constitucional fundada en la definición del principio de la igualdad básica de los sexos. Cuando decimos "igualdad básica de los sexos", ello implica aceptar, al mismo tiempo, la existencia obvia de diferencias entre ellos, sean éstas biológicas o psicológicas, pero que se complementan en las funciones sociales. (...) Nuestra gran inquietud es que la implementación de una política relativa a la mujer ayude a un más ponderado desarrollo de la familia, base del equilibrio, de la armonía social y de la paz" (DSCS N16, 27/11/1990:18).

Lo anterior, corresponde a un extracto del discurso de la Senadora Carmen Frei Ruiz Tagle, militante $\mathrm{DC}^{9}$, del cual forma parte el propio presidente. Este discurso fue realizado en la sesión del Proyecto de Ley en que se discutió la creación del "SERNAM".

\subsection{Las paradojas del "SERNAM" y su articulación con el modelo económico de Chile}

Analizar de qué forma se materializaron las demandas feministas con la agenda política del Gobierno de Aylwin y además caracterizar los programas instaurados para

\footnotetext{
8 Extraído de la página web del Servicio Nacional de la Mujer: http://www.minmujeryeg.gob.cl/sernameg/

9 Partido político "Democracia Cristiana", tendencia centro 
entender cómo se articularon al modelo económico del país, nos permite evidenciar paradojas en el proceso.

De acuerdo a las Metas Ministeriales del año $1991^{10}$, relacionadas a políticas de género, el Estado propone reformar el régimen patrimonial para otorgar plena capacidad de administración de bienes a las mujeres, la creación de un Tribunal de Familia, la creación de una Ley de Violencia Intrafamiliar y la derogación de normas que prohíben el trabajo de la mujer, reforzamiento a la fiscalización de las condiciones sanitarias y de seguridad en el trabajo, permiso parental en caso de nacimiento de hijos, en complemento del permiso maternal y la creación de un instrumento público que regule el trabajo femenino en casas particulares.

También se compromete a la realización de un Plan Nacional de Apoyo a la Mujer Jefa de Hogar, destinado a mejorar la calidad de vida de 100.000 mujeres jefas de hogar en condiciones de extrema pobreza; prevenir el embarazo adolescente y la creación de Centros de Información, que orienten a las mujeres en sus derechos y a los beneficios sociales a los que pueden acceder.

Mediante los proyectos expuestos, se toma la medida administrativa de que en 1991 se pondrá en funcionamiento el "SERNAM", siendo María Soledad Alvear Valenzuela, militante DC y ajena al movimiento de mujeres, designada como Ministra Directora ${ }^{11}$. Sobre la estructura interna del "SERNAM", El Reglamento Interno de $1991^{12}$ reconoce 11 niveles de organización, encabezadas por la Dirección Nacional, seguida por la Subdirección Nacional, la Fiscalía, el Departamento de Desarrollo Regional, el Departamento de Planificación y Estudios, el Departamento de Administración y Finanzas, el Departamento de Comunicaciones, el Departamento de Cooperación y Relaciones Internacionales, el Departamento de Relaciones Públicas, el Departamento de Capacitación y las Direcciones Regionales.

Sobre lo último, es importante mencionar que, si bien los programas están dispuestos desde el "SERNAM", a nivel municipal serán principalmente mujeres profesionales o técnicos del área social, quienes, a través de un contrato de tipo honorarios, desarrollarán la labor de llevar a cabo los programas de género a la población. Éstas no son mencionadas ni reconocidas como trabajadoras del servicio, resultando paradójico, en el sentido de que el "SERNAM" se propone el resguardo de las mujeres trabajadoras, pero emplea mediante contratos de trabajos precarios a las mujeres encargadas de llevar a cabo estos programas (Schild, 2000).

Otra arista importante de repensar son las bases generales para la creación del "SERNAM". A pesar de que la "CNMD" planteaba la necesidad de problematizar la situación de las mujeres en términos estructurales, solo se aborda por Estado la

\footnotetext{
${ }^{10}$ Extraído de página web: http://www.archivospublicos.cl/index.php/metas-ministeriales-1997

${ }^{11}$ Extraído de página web: http://www.archivospublicos.cl/index.php/aceptacion-cargo-de-directornacional-sernam-rango-ministro-de-estado

${ }^{12}$ Extraído de página web: http://www.archivospublicos.cl/index.php/reglamento-interno-del-servicionacional-de-la-mujer
} 
situación de precariedad económica de las mujeres más pobres, por lo que las demandas relativas a participación política y a la sexualidad, quedan fuera del quehacer institucional. Franceschet (2003) indica que esto se genera por la sola contemplación de los intereses de género enmarcados en los objetivos del Estado, generándose así y tal como plantea Fraser (1989), la disputa de marcos de sentido para entender las problemáticas de las mujeres.

A propósito de esto, diversas investigaciones (Montecinos y Rossetti, 1990; Álvarez, 2011; Godoy, 2013; Guzmán, 1997; Baldez, 1999; Schild, 2015-2016) exponen sobre lo problemática que significó para el gobierno de Aylwin la creación de este servicio público. La inclusión de la palabra género en el lenguaje institucional, significó la reticencia de los sectores conservadores de derecha y también desde algunos partidos políticos que apoyaban el gobierno democrático, los que veían como amenaza a la familia que el Estado se inmiscuya en temáticas de índole privado y que se haga referencia a la igualdad entre hombres y mujeres. Sostenían además que la palabra género era una amenaza por su impronta feminista. Cabe destacar también, que, de acuerdo a las intervenciones de los Senadores, en dónde se discutió acerca de la creación del "SERNAM", no se hizo señalamiento al rol del movimiento feminista en la consecución de que se legisle sobre la condición de la mujer en Chile, refiriéndose además a estas como las encargadas del bienestar de sus hijos y familia, por lo que el "SERNAM", en sus orígenes legislativos corresponde más a una institución pública destinada a la familia que a subsanar las desigualdades de las mujeres.

Las investigaciones también dan cuenta de cómo se terminó por fragmentar el movimiento feminista. Las mujeres pertenecientes a las esferas influyentes de los partidos de izquierda y las feministas universitarias de clase media y alta, fueron las que ocuparon los puestos en la institucionalidad y las mujeres de sectores populares pasaron a ser las usuarias de los programas (Waylen, 1997; Godoy, 2013; Guzmán, 1997; Baldez, 1999; Ríos et.al., 2003) o las "clientas de programas estatales", como indica Schild (2015-2016).

Las discrepancias entre feministas o femócratas ${ }^{13}$, (Valdés y Fernández, 2006) fue el más controversial y aunque ambos sectores manifestaban la necesidad de recuperar la democracia y combatir el sexismo, disentían en la forma de conseguir tales objetivos. Por un lado, las "feministas", abogaban por la realización de una agenda de género con el apoyo de un conglomerado político independiente, sostenían además que las "políticas", ponían por sobre de las demandas el movimiento feminista y el combate contra la subordinación femenina, las estrategias de los partidos en los que militaban. Y, por otra parte, las "políticas" apelaban a que no existía una incompatibilidad su doble militancia, puesto que creían que la democracia es la condición básica para combatir las desigualdades de género y que el pertenecer a los partidos políticos se convertía en una oportunidad para favorecer la creación de

13 "Femócratas" se refiere a la mezcla entre el término feministas y burócratas. 
reformas desde el gobierno (Ríos et al., 2003). Esta división acentuaría el declive que sufrió el movimiento al restaurarse la democracia.

De acuerdo a esto último, Baldez (1999) analiza que el impacto del movimiento feminista sobre la agenda pública del gobierno se puede también explicar en función de la naturaleza competitiva de los partidos políticos. No se trata de que el retorno per se de los partidos hace que los movimientos declinen, sino que la lucha por el poder entre estos los impulsa a tolerar ciertos tipos de movilización y sofocar otros. Así, la facción conservadora del bloque democrático, buscó proteger sus intereses y limitar la competencia entre sus contrapartes más progresistas que más afinidad tenían con la "CNMD". Así, la agenda de género instaurada en el gobierno optó por recuperar el control de la agenda política de las mujeres, visualizándolas como electorado leal a su partido, y limitando los alcances de los partidos de izquierda.

Waylen (1995) sostiene que el bloque democrático aceptó retomar sólo aquellas medidas destinadas a proteger la familia. Para Valenzuela (1993), la razón por la que las feministas y el Estado no coincidieron en la práctica, es porque quienes formaban parte de la "CNMD" asumieron que el gobierno, y por implicación el Estado, era de género neutral, por lo que comprometerse con el Estado sería un proceso sencillo para lograr la expansión de los derechos, supuesto que en la práctica resultó problemático.

De acuerdo a los programas del "SERNAM", indica Schild (2015) que estos buscan convertir a las beneficiarias en personas que puedan subsanar su condición de precariedad y vulnerabilidad y hacerse responsables de su propio desarrollo. Si bien los programas establecidos forman parte de las demandas de la "CNMD", responde a un proceso de cambios culturales asociados al proyecto neoliberal.

Sobre esto último, es importante retomar también lo relacionado al contexto económico al momento de la creación de esta institución. Así, es necesario remontarnos a los inicios movimiento feminista en Chile, en un período de la historia en el cual el país sufrió una serie de reformas económicas y sociales que terminó con la instauración de la Junta Militar presidida por Pinochet. En este contexto, se ponen en marcha medidas neoliberales bajo la asesoría de la Escuela de Chicago, en donde Milton Friedman y los Chicago Boys, orientaron a Pinochet en la reforma de políticas económicas que permitieran la apertura económica al comercio internacional, bajo la instauración de una nueva estructura productiva (Garretón ,1988; Klein, 2012)

Se planteó que la mejor forma de recuperar el equilibrio económico y social era dejando que el mercado funcionara en absoluta libertad. Así, desde el año 1974, las políticas públicas favorecieron que los empresarios mantuvieran actividad económica orientada al mercado y la concentración del poder en un número restringido de grupos económicos de gran envergadura, manteniendo para esto un fuerte marco represivo estatal. Esto trajo aparejado hasta los años 80 un aparente crecimiento económico, basado en el endeudamiento externo del Estado y un alto movimiento especulativo de capital (Garretón, 1988). 
Las consecuencias de este modelo en términos de desigualdad social, significaron una desarticulación de la relación clásica entre Estado y Sociedad Civil y una fuerte desindustrialización (Garretón, 1988) que condujo a una mayor pobreza e inequidad, por lo que Chile, además de ser golpeado por la muerte de cientos de personas, también vio engrosar las filas de desempleados y poblaciones precarizadas. Entonces, a pesar de la transición a un gobierno democrático, se mantuvieron las políticas económicas instauradas en la dictadura.

Sobre esto, Fermandois (2007) indica que en los años noventa los gobiernos de la Concertación de Partidos por la Democracia en Chile reafirmaban y elogiaban las reformas impulsadas por el régimen militar y sus asesores económicos, asumiendo las estrategias económicas neoliberales, pero dando un contenido social a estas. En ese sentido, el éxito de la implantación en dictadura y permanencia en los gobiernos democráticos de la ideología neoliberal, debe quizás su éxito a la generalización de la idea de que no existe un modelo alternativo posible, por lo que sólo es viable introducir correcciones (Garretón, 2012). Así, la Concertación de Partidos por la Democracia además de enfrentarse al desafío de restablecer la democracia, tuvo que enfrentarse a un modelo social y económico consolidado y que había sido impuesto sin restricciones, dándose el fenómeno que Drake (2009) Ilama "democracia neoliberal".

\section{Conclusiones}

Sostengo que la institucionalidad de género en Chile, si bien responde a una necesidad politizada de la "CNMD", se ajusta estratégicamente a subsanar la condición de precariedad del sector de mujeres más pobres del país, para hacerlas útiles al modelo económico imperante y a la vez las constituye como sujetos de políticas de género, dejando entonces por fuera de esta categorización a todas las mujeres que no cumplen con los requisitos de focalización. Así y tal como plantean Guzmán y Montaño (2012) en esta investigación se considera que las transformaciones en las relaciones de género no son sólo producto de la voluntad y la acción de los actores, sino también de las oportunidades y restricciones que ofrecen las normas institucionales que regulan las relaciones entre sujetos. Para este caso, no sólo se remite a las normas institucionales, sino también a los propios discursos y quehacer gubernamentales, a los partidos políticos, a lo que sin dudas se debe agregar, cómo el contexto económico moldea también la acción estatal.

También, es posible entender las políticas de género como espacios de intersección entre el Estado y diversos actores sociales. Así, estos espacios de negociación y conflicto, para el caso chileno, significaron la sola contemplación de las demandas feministas referentes a subsanar la precariedad económica de las mujeres más pobres, en desmedro de los requerimientos de índole político, de participación y de derechos sexuales, provocando además la fragmentación del movimiento feminista. 
En relación a cómo se articuló la creación del "SERNAM" con las demandas feministas de la "CNMD", se puede establecer que el movimiento de mujeres fue un actor clave y decisorio para salir de la dictadura militar. El movimiento establece en general las necesidades de las mujeres y la facción "CNMD" conformada por mujeres con capital político, social, cultural y económico más alto se encargan de negociar con los partidos políticos de centroizquierda sobre la creación de una institución que ejecute políticas de género. Las mujeres que no formaban parte de este grupo pasaron a ser las usuarias o clientas de los programas, fragmentando los cimientos del movimiento

Otro aspecto analítico a destacar es cómo la institucionalidad de género se relaciona con el contexto económico del país, que, como revisamos, desde la dictadura militar atravesó por ajustes estructurales que facilitaron la neoliberalización de la economía, del mercado y la acción estatal. La sola contemplación de las necesidades económicas de las mujeres, condicionó la reactivación financiera de la parte más pobre de la población, permitiendo mediante la acción del "SERNAM" la habilitación de estas para su posterior inserción al mercado laboral, creando sujetos de intervención en quienes cumplían con los criterios de focalización, pero también sujetos que no son parte de las políticas de género. Estos sujetos son dinámicos, responden al contexto político, sociocultural y económico y se encuentran atravesados por la articulación de diversas desigualdades sociales, en donde confluyen distintos marcos de sentido y relaciones de poder.

\section{Bibliografía}

Álvarez, Sonia (1999): "Advocating feminism: The Latin American Feminist NGO 'Boom', en International Feminist Journal of Politics, N 1:2, p. 181-209, [On Line]. Recuperado de: https://edisciplinas.usp.br/pluginfile.php/4304601/mod_resource/content/1/alvar ez1999.pdf

Anzaldúa, Gloria (1999): Borderlands. La frontera. The new meztiza. Aunt Lute Books.

Argüello, Sofía (2013): "El proceso de politización de la sexualidad: identificaciones y marcos de sentido de la acción colectiva", en Revista Mexicana de Sociología, No 75, p. $173-200$.

Baldez, Lisa (1999): "La política partidista y los límites del feminismo de estado en Chile", en Jaksic, Iván y Drake Paul (compiladores): El modelo chileno. Democraciay desarrollo, p. 407-433. Chile: LOM.

Bauman, Zygmunt (1999): La globalización. Consecuencias humanas. Argentina: Fondo de Cultural Económica.

Beauvior, Simone ([1949] 1999): El segundo sexo. Buenos Aires: Editorial Sudamericana.

Benería, Lourdes (1999): "Mercados globales, género y el hombre de Davos", en Revista La Ventana, No 10, p. 7-48. 
Bourdieu, Pierre (1997): Razones prácticas. Sobre la teoría de la acción. Barcelona: Anagrama.

Diario de Sesiones de la Cámara del Senado. Legislatura $321^{a}$, Extraordinaria. Sesión 16a , en martes 27 de noviembre de 1990. Ordinaria, p.18. Recuperado de: file:///C:/Users/HP/Downloads/diario 25051\%20(1).pdf

Drake, Paul (2009): Between tyranny and anarchy. A history of democracy in latin america, 1800-2006. Stanford University Press.

Fermandois, Joaquín (2007): "Modernización, desarrollo, dictadura: el papel de Sergio de Castro", en Centro de Estudios Públicos, No 108, p. 281-313 [On Line]. Recuperado de: http://www.memoriachilena.gob.cl/6o2/w3-article86237.html

Foucault, Michel (1999): Ética, estética y hermenéutica. Editorial Paidos Ibérica.

Foucault, Michel (2007): Nacimiento de la biopolítica. Curso en el Collegé de France (1978 1979). Buenos Aires: FCE.

Franceschet, Susan (2003): "States and women's movements: the impact of Chile's Servicio Nacional de la Mujer on women's activism", en Latin American Research Review, Vol. 38, No 1, p. 9-40 [On Line]. Recuperado de: https://www.jstor.org/stable/1555433?seq=1\#page_scan_tab_contents

Fraser, Nancy (1989): Unruly practice, power, discourse and gender in contemporary social theory. Minneapolis: University of Minnesota Pres.

Fraser, Nancy (1997): Iustitia Interrupta. Reflexiones críticas desde la posición postsocialista. Siglo del Hombre Editores: Universidad de Los Andes.

Fraser, Nancy (2017): Fortunas del feminismo. Del capitalismo gestionado por el Estado a la crisis neoliberal. Quito, Ecuador: IAEN.

García, Evangelina (2008): Políticas de Igualdad, Equidad y Gender Mainstreaming. ¿De qué estamos hablando?: Marco Conceptual. PNUD.

Garretón, Manuel (1988): "El Plesbicito de 1988 y la transición a la democracia", en Cuadernos de Difusión FLACSO Chile, [On Line]. Recuperado de: http://flacsochile.org/biblioteca/pub/publicos/1988/libro/000017.pdf

Girón, Alicia (2009): "Género, globalización y desarrollo", en Girón, Alicia (coordinadora): Género y Globalización, p. 77-97. Buenos Aires: CLACSO.

Godoy, Carmen (2013): "El estado chileno y las mujeres en el siglo XXI. De los temas de la mujer al discurso de la igualdad de géneros", en Diálogos Revista Electrónica, Vol. 14, $\mathrm{N}^{\circ}$ 1, p. 97-123 [On Line]. Recuperado de: https://www.scielo.sa.cr/scielo.php?script=sci abstract\&pid=S1409469X20130 0010 $\quad 004 \& \operatorname{lng}=e n \& n r m=i s 0 \&$ tlng=es

Guzmán, Virginia y Montaño, Sonia (2012): Políticas públicas e institucionalidad de género en América Latina (1985-2010). CEPAL.

Guzmán, Virginia (1997): "La equidad de género como tema de debate y de políticas públicas", en: Largo, Eliana (editora): Género en el Estado. Estado del Género, p.

55 70. Chile: Isis Internacional.

Harvey, David (2007): Breve historia del neoliberalismo. Ediciones Akal. 
Herrera, Gioconda (2001): "El género en el Estado: entre el discurso civilizatorio y la ciudadanía", en Revista Iconos, N¹1, p. 80-88.

Hill Collins, Patricia (2012): "Rasgos distintivos del pensamiento feminista negro", en Fabardo, Mercedes (editora): Feminismos negros. Una antología, p. 99-134. Traficantes de sueños.

Kirkwood, Julieta (1986): Ser Política en Chile. Las Feministas y los Partidos. FLACSO Chile.

Klein, Naomi (2012): La doctrina del shock. El auge del capitalismo del desastre. Editorial Booket.

Lind, Amy (2005): Gendered paradoxes. Women's Movements, state restructuring, and global development in Ecuador. Penn State University Press.

Lorde, Audre (2002): La hermana, la extranjera. Editorial Horas y Horas.

Mahmood, Saba (2008): "Teoría feminista y el agente dócil: algunas reflexiones sobre el renacimiento islámico en Egipto", en Hernández, Rosalva y Suárez, Liliana (coordinadoras): Descolonizando el feminismo. Teorías y prácticas desde los márgenes, p. 165-222. Cátedra Ediciones.

Molyneux, Maxine (2008): "Justicia de género, ciudadanía y diferencia en América Latina", en Prieto, Mercedes (editora): Mujeres y escenarios ciudadanos, p. 22-56. Quito: FLACSO.

Montecino, Sonia y Rosetti, Josefina (1990): Tramas para un nuevo destino: propuestas de la Concertación de Mujeres por la Democracia. Santiago: Arancibia Hnos.

Murillo, Susana (2011): "Estado, sociedad civil y gubernamentalidad neoliberal", en Revista de la Carrera de Sociología, Vol.1, № 1, p. 91-108.

Ríos, Marcela; Godoy, Lorena y Guerrero, Elizabeth (2003): ¿Un nuevo silencio feminista? La transformación de un movimiento social en el Chile posdictadura. Chile: CEM y Ediciones cuarto propio.

Rodríguez, José (2003): "La producción de la subjetividad en los tiempos del neoliberalismo: hacia un imaginario con capacidad de transformación social", en Cuadernos de Relaciones Laborales, N¹, p. 89-105.

Rousseau, Stéphanie (2012): Mujeres y ciudadanía. Las paradojas del neopopulismo en el Perú de los noventa. Lima: Instituto de Estudios Peruanos.

Schild, Verónica (2000): Equidad de género sin justicia social: los derechos de la mujer en la era neoliberal [On Line]. Disponible en: https://nacla.org/search/node/schild

Schild, Verónica (2015): "Los feminismos y la construcción del estado social neoliberal en América Latina", en Enríquez, Narda et al. (edit): Desigualdades en un mundo globalizado. Lima: CISEPA; Desigualdades.net.

Schild, Verónica (2016): "Feminismo y Neoliberalismo en América Latina", en Revista Nueva Sociedad, No 265 [On Line]. Recuperado de: https://nuso.org/articulo/feminismo-y-neoliberalismo-en-america-latina/

Scott, Joan (2008): Género e historia. Fondo de Cultura Económica Universidad Autónoma de la Ciudad de México. 
Valdés, Teresa y Fernández, María (2006): "Género y política: un análisis pertinente", en Revista de Ciencia Política, Vol. 46, p. 9-33 [On Line]. Recuperado de: https://revistapolitica.uchile.cl/index.php/RP/article/view/17007

Valdés, Teresa (1987): Las mujeres y la dictadura militar en Chile. Chile: FLACSO.

Valenzuela, María (1993): "Las mujeres en la transición democrática", en Drake, Paul y Jaksic, Iván (editores): El difícil camino hacia la democracia en Chile 1982-1990, p. 307-350. Chile: FLACSO.

Wacquant, Louis (2003): "Tres pasos hacia una antropología del neoliberalismo real" [On Line]. Recuperado de: https://biblat.unam.mx/es/revista/herramientabuenos aires/articulo/tres-pasos-hacia-una-antropologia-historica-delneoliberalismo-real

Wappenstein, Susana y Villamediana, Virginia (2014): "Estudio regional de las políticas públicas en el eje de género", en Bonilla, Adrián et al. (editores) Políticas sociales en América Latina y el Caribe, escenarios contemporáneos, inversiones y necesidades, p. 283-311: FLACSO Ecuador.

Waylen, Georgina (1995): "Women's movements, the state and democratization in Chile", en IDS Bulletin, Vol. 23, Na 3, p. 86-93 [On Line]. Recuperado de: https://onlinelibrary.wiley.com/doi/10.1111/j.1759-5436.1995.mp26003010.x

Waylen, Georgina (1997): "'Womens movements the state and democratization in Chile: the establishment of SERNAM"'", en Geotz, Anne (ed.): GettingInstitutions Right for Development, p. 90-104 London: Zed Books. 


\section{Cómo referenciar este artículo/How to reference this article:}

Orellana, P. (2020). La construcción de sujetos en torno a la institucionalización de políticas públicas de género en Chile. iQUAL. Revista de Género e lgualdad, 3, 108-131, doi: 10.6018/iqual.394251

Orellana, P. (2020). La construcción de sujetos en torno a la institucionalización de políticas públicas de género en Chile. [The construction of subjects around in the processes of institutionalization of public gender policies in Chile]. iQUAL. Revista de Género e Igualdad, 3, 108-131, doi: 10.6018/iqual.394251 